Old Dominion University

ODU Digital Commons

Bioelectrics Publications

Frank Reidy Research Center for Bioelectrics

$5-2016$

\title{
A 'Tissue Model' to Study the Barrier Effects of Living Tissues on the Reactive Species Generated by Surface Air Discharge
}

Tongtong He

Dingxin Liu

Han Xu

Zhichao Liu

Dehui Xu

See next page for additional authors

Follow this and additional works at: https://digitalcommons.odu.edu/bioelectrics_pubs

Part of the Biomedical Commons, Biophysics Commons, Molecular, Cellular, and Tissue Engineering Commons, and the Physics Commons

\section{Repository Citation}

He, Tongtong; Liu, Dingxin; Xu, Han; Liu, Zhichao; Xu, Dehui; Li, Dong; Li, Qiosong; Rong, Mingzhe; and Kong, Michael G., "A 'Tissue Model' to Study the Barrier Effects of Living Tissues on the Reactive Species Generated by Surface Air Discharge" (2016). Bioelectrics Publications. 220.

https://digitalcommons.odu.edu/bioelectrics_pubs/220

\section{Original Publication Citation}

He, T. T., Liu, D. X., Xu, H., Liu, Z. C., Xu, D. H., Li, D., ... Kong, M. G. (2016). A 'tissue model' to study the barrier effects of living tissues on the reactive species generated by surface air discharge. Journal of Physics D: Applied Physics, 49(20). doi:10.1088/ 0022-3727/49/20/205204 


\section{Authors}

Tongtong He, Dingxin Liu, Han Xu, Zhichao Liu, Dehui Xu, Dong Li, Qiosong Li, Mingzhe Rong, and Michael G. Kong 
PAPER

\section{A 'tissue model' to study the barrier effects of living tissues on the reactive species generated by surface air discharge}

To cite this article: Tongtong He et al 2016 J. Phys. D: Appl. Phys. 49205204

View the article online for updates and enhancements.

\section{Related content}

A novel large-scale plasma source: two
$\frac{\text { discharge modes and their correlation to }}{\text { the production of aqueous reactive }}$
species
Bingchuan Wang, Dingxin Liu, Zhiquan
Zhang et al.
- How to assess the plasma delivery of
RONS into tissue fluid and tissue
Jun-Seok Oh, Endre J Szili, Nishtha Gaur
et al.
- Physicochemical processes in the indirect
interaction between surface air plasma
and deionized water
Z C Liu, D X Liu, C Chen et al.

\section{Recent citations}

- A comparative study on the transdermal
$\frac{\text { penetration effect of gaseous and aqueous }}{\text { plasma reactive species }}$
Xin Liu et al
- Investigation of non-thermal plasma
$\frac{\text { effects on lung cancer cells within 3D }}{\text { collagen matrices }}$
Surya B Karki et al
- The assessment of cold atmospheric
plasma treatment of DNA in synthetic
$\frac{\text { models of tissue fluid, tissue and cells }}{\text { Endre J Szili et al }}$

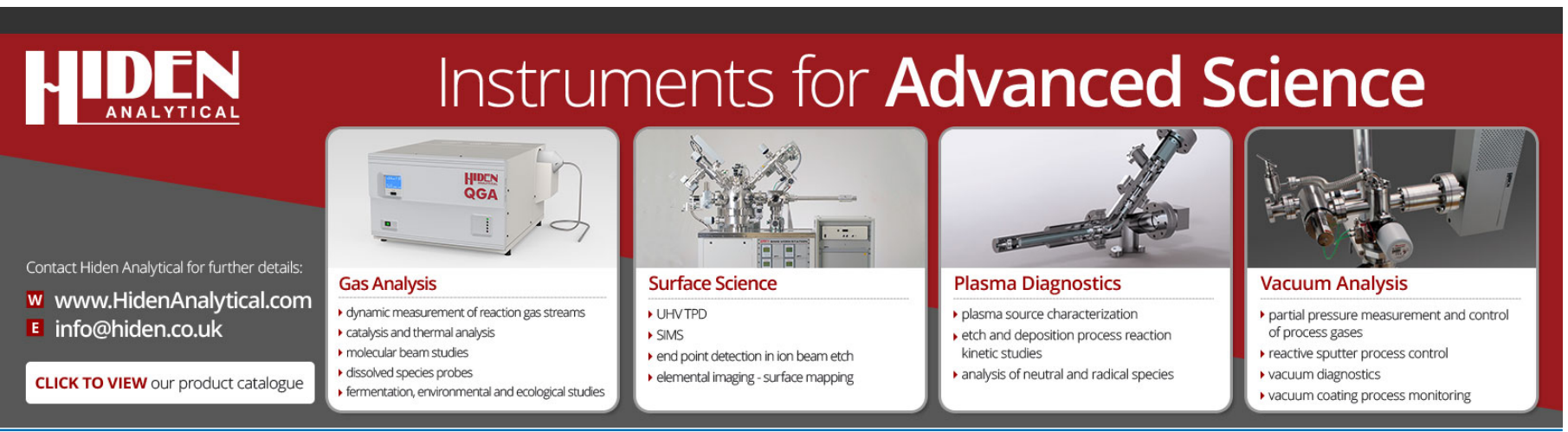




\title{
A 'tissue model' to study the barrier effects of living tissues on the reactive species generated by surface air discharge
}

\author{
Tongtong $\mathrm{He}^{1}$, Dingxin Liu ${ }^{1}$, Han $\mathrm{Xu}^{1}$, Zhichao liu ${ }^{1}$, Dehui $\mathrm{Xu}^{1}$, Dong $\mathrm{Li}^{1}$, \\ Qiaosong $\mathrm{Li}^{1}$, Mingzhe Rong ${ }^{1}$ and Michael G Kong ${ }^{1,2,3}$ \\ ${ }^{1}$ State Key Laboratory of Electrical Insulation and Power Equipment, Center for Plasma Biomedicine, \\ Xi'an Jiaotong University, Xi' an 710049, People's Republic of China \\ 2 Frank Reidy Center for Bioelectrics, Old Dominion University, Virginia 23508, USA \\ ${ }^{3}$ Department of Electrical and Computer Engineering, Old Dominion University, Virginia 23529, USA \\ E-mail: liudingxin@mail.xjtu.edu.cn and mglin5g@gmail.com
}

Received 19 January 2016, revised 17 March 2016

Accepted for publication 18 March 2016

Published 18 April 2016

\begin{abstract}
Gelatin gels are used as surrogates of human tissues to study their barrier effects on incoming reactive oxygen and nitrogen species (RONS) generated by surface air discharge. The penetration depth of nitrite into gelatin gel is measured in real time during plasma treatment, and the permeabilities of nitrite, nitrate, $\mathrm{O}_{3}$ and $\mathrm{H}_{2} \mathrm{O}_{2}$ through gelatin gel films are quantified by measuring their concentrations in the water underneath such films after plasma treatment. It is found that the penetration speed of nitrite increases linearly with the mass fraction of water in the gelatin gels, and the permeabilities of nitrite and $\mathrm{O}_{3}$ are comparably smaller than that for $\mathrm{H}_{2} \mathrm{O}_{2}$ and nitrate due to differences in their chemistry in gelatin gels. These results provide a quantitative basis to estimate the penetration processes of RONS in human tissues, and they also confirm that the composition of RONS is strongly dependent on the tissue depth and the plasma treatment time. A small electric field of up to $20 \mathrm{~V} \mathrm{~cm}^{-1}$ can greatly reduce the barrier effects of the tissue model regardless of their directions, for which the underlying mechanism is unclear. However, the electric field force on the objective RONS should not be the dominant mechanism.
\end{abstract}

Keywords: cold atmospheric-pressure plasma, surface air discharge, tissue model, barrier effect, RONS

(Some figures may appear in colour only in the online journal)

\section{Introduction}

The interaction between cold atmospheric-pressure plasmas and living tissues is a critical topic of plasma science and many clinical applications such as chronic ulcer therapy [1], blood coagulation [2], dental care [3] and cancer treatment [4]. Physically, living tissue acts as a barrier to inhibit the penetration of plasma-generated 'cocktails', including the reactive oxygen and nitrogen species (RONS) which are believed to be responsible for the therapeutic effects [5]. Considering that some RONS have very short lifetimes in living tissues, $\sim 1 \mathrm{~ns}$ in the case of $\mathrm{OH}$ for instance, the composition of plasma-induced RONS should be strongly dependent on the tissue depth. This indicates that the RONS in living tissues which are effective for clinical applications might be very different to that existing in the nearby gas phase. For example, melanoma cells normally distribute in the hypodermis layer which is at least $100 \mu \mathrm{m}$ from the skin surface [6,7], preventing most kinds of gaseous reactive species from penetrating through the skin layer before reaching the target spots. So far, little has been known of the barrier effects of living tissues on the plasma-generated RONS, leading to a big challenge for designing and optimizing plasma sources for clinical applications. 
Studying the barrier effects requires quantification of the penetration depths of RONS in human tissues at a microscale level, which is beyond the current capability of experimental investigations. Tissue models are used for this investigation, of which the simplest one is water [8]. This is because the living tissues contain a large amount of water; for example, the mass fraction of water in human skin is $\sim 70 \%$ [9]. So, the penetration of RONS in water is more or less similar to that in living tissues. Many studies have been reported on plasma-water interactions in recent years, but only a few of them quantified the penetration depths of RONS by simulation [8-11]. This is mainly due to the liquidity of water and the irregular activity of aqueous molecules which lead to a big challenge for spatially resolved measurements [12, 13]. Recently, gelatin gel has been used by Szili et al as a surrogate for real tissue to study the penetration of RONS generated by a plasma jet [14-16]. The gelatin gel consists of water and collagen, the most abundant extracellular matrix protein, and the collagen inhibits the liquidity of water. This suggests that properly prepared gelatin gels can serve as a good tissue model for studying the barrier effects of human tissues on plasma-generated RONS.

In this paper, tissue models made of gelatin gels are used to investigate their barrier effects on the RONS generated by a surface air discharge. Surface air discharge easily generates large-area, high-reactivity cold plasmas, and hence it has recently been used in various biomedical applications [17-20]. Different to the reactive species generated by plasma jets as reported by Szili et al $[15,16,21,22]$, for surface air discharge the charged species cannot act on the tissue models because of the air gap between the plasmas and the gelatin gels which are filled in dishes. The biological effects have been found to be very different for the charged species acting on the targets or not [23]. Moreover, the compositions of neutral RONS are very different for a helium plasma jet and a surface air plasma; for example, the yield of $\mathrm{O}_{3}$ by the surface air plasma is typically much larger [24, 25]. These findings suggest that the tissue models treated by a surface air discharge should be very different to those treated by a helium plasma jet. The barrier effects of the tissue models are quantified by online measuring the penetration depths of nitrite $\left(\mathrm{HNO}_{2} / \mathrm{NO}_{2}^{-}\right)$ during the plasma treatment, as well as comparing the densities of nitrite, nitrate $\left(\mathrm{HNO}_{3} / \mathrm{NO}_{3}^{-}\right), \mathrm{O}_{3}$ and $\mathrm{H}_{2} \mathrm{O}_{2}$ in dishes of deionized water with and without the covering of gelatin gel films after plasma treatment. For the purpose of reducing the barrier effects, the influence of small electric fields on the barrier effects is then studied. Concluding remarks are presented at the end.

\section{Experimental methods}

The experimental setup for online measurement of nitrite penetration in tissue models is shown in figure 1. It consists of a surface discharge reactor and a downstream dish of gelatin gels, separated from each other by an air gap of $1 \mathrm{~cm}$. The discharge reactor consists of a plane high-voltage electrode, a grounded mesh electrode, and a dielectric sheet sandwiched between the two electrodes. Each grounded mesh has a hexagon shape. For more details of the discharge reactor, please refer to our previous publications [11, 26-28]. Gelatin gels were prepared by mixing pure gelatin powder (MP biomedicals) with water solution according to the standard protocol. The water solution was made by adding Griess reagent (beyotime biotechnology) into deionized water for indicating nitrite by color-forming reactions. The mass fraction of gelatin gel is varied from $1.5 \%$ to $30 \%$ to represent different kinds of living tissues; for example, the mass fraction of water in human skin is typically $70 \%$ (equivalent to $30 \%$ of gelatin), and for blood which is considered a specialized form of connective tissue it is $\sim 85 \%$ [29]. The dish filled with gelatin gel was tailor-made of quartz glass, which has a cuboid shape with dimensions of $2 \times 3 \times 1.2 \mathrm{~cm}$ (width $\times$ length $\times$ height) The thickness of each side is $0.1 \mathrm{~cm}$, so the height of gelatin gel is kept at $1.3 \mathrm{~cm}$. Compared to the cylinder shape which is typically used for Petri dishes, the cuboid shape is important in this study because it reduces the refraction of light at the dish side and hence benefits the absorption spectroscopy measurements. The discharge reactor and the dish of gelatin gel were semi-closed in a box, of which the height position was controlled by a height adjuster with an adjusting precision of $10 \mu \mathrm{m}$ (see figure 1).

For the online measurements of nitrite penetration, a sinusoidal voltage with a peak-to-peak value of $9 \mathrm{kV}$ and a frequency of $20 \mathrm{kHz}$ was applied. The waveforms of discharge voltage and current were recorded by an oscilloscope (Tektronix, DPO3000) with a high-voltage probe (Tektronix, P6015A) and a current probe (Tektronix, P6021). Surface air plasmas were generated in the grounded meshes, covering a large area of about $16 \mathrm{~cm}^{2}$ with a good mesh-to-mesh homogeneity [11]. Gaseous reactive plasma species diffuse through the air gap and some of them penetrate into the gelatin gels. For the absorption spectroscopy of the gelatin gels, a laserdriven light source (Energetiq, EQ-99FC) was used with spectral output from 170 to $2100 \mathrm{~nm}$. The light was vertically incident on one side of the dish, and the emergent light out of the opposite side was collected by a spectrometer (Ocean Optics, USB2000). The optical fibers used here have a diameter of $60 \mu \mathrm{m}$, so the spatial resolution should be $\sim 60 \mu \mathrm{m}$. However, the diffraction and interference of light at the gasgelatin gel interface are very strong, and hence it is difficult to determine the interface position. To avoid this, two homemade field stops were used in front of the optical fibers (see figure 1). Each field stop is a hollow tube which has an inner diameter of $1 \mathrm{~mm}$ and a length of $2 \mathrm{~cm}$, and therefore only light almost parallel to the axis of the tube can pass through the field stop. The field stops were fixed, and hence the light could pass though different depths of the gelatin gel by adjusting the height adjuster. Based on the experimental setup shown in figure 1, the penetration depths of nitrite were detected by comparing the absorption spectral lines of the gelatin gels before and after plasma treatments.

For the offline (after plasma treatment) measurements of nitrite, nitrate, $\mathrm{H}_{2} \mathrm{O}_{2}$ and $\mathrm{O}_{3}$ in the deionized water covered by $1 \mathrm{~mm}$ thick gelatin gel films, most of the experimental conditions were the same as that for the online measurement. 


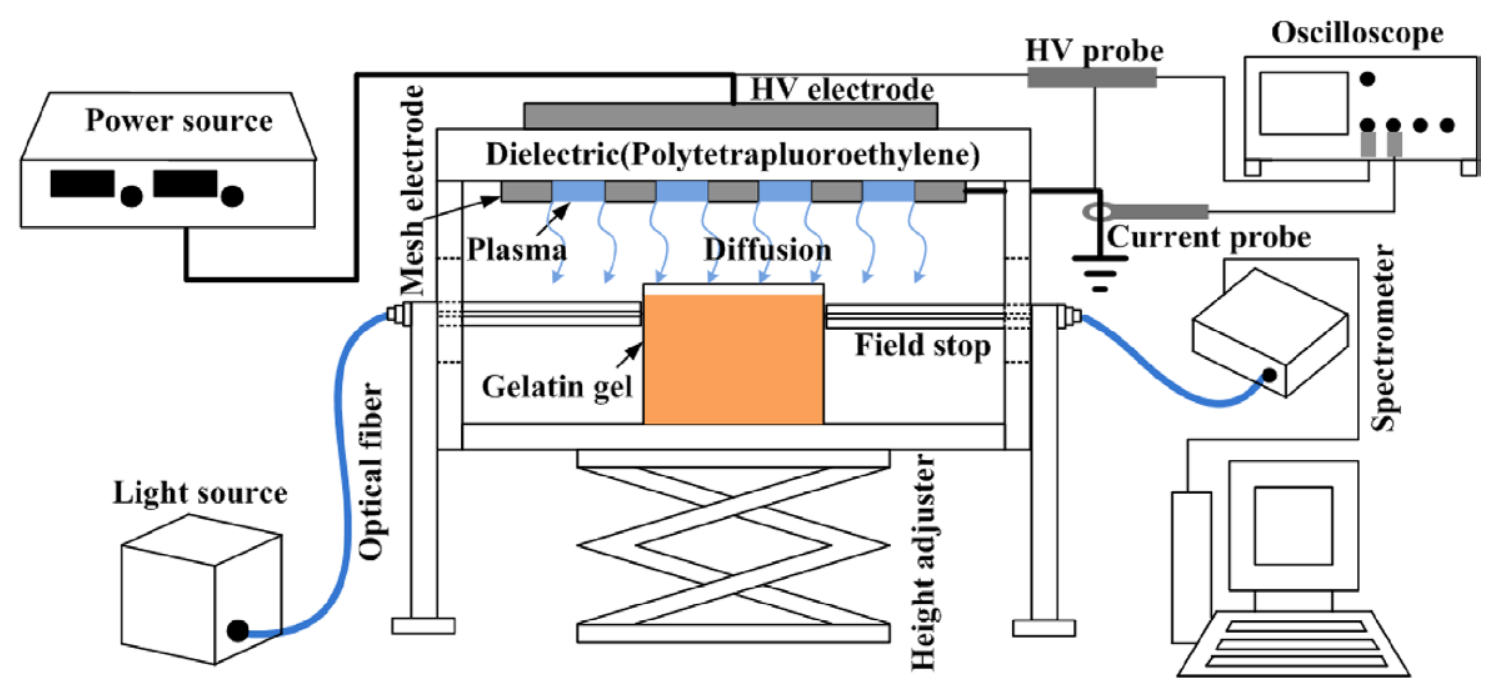

Figure 1. Schematic diagram of the experimental setup.

The main differences are that absorption spectroscopy was no longer used, the deionized water filled in 96-well plates was covered by gelatin gel films, and the RONS in the water were detected by spectrophotometry immediately after plasma treatments. The concentrations of $\mathrm{H}_{2} \mathrm{O}_{2}$, nitrate and nitrite were measured by using a microplate reader (Thermo Scientific Varioskan ${ }^{\circledR}$ Flash Reader). Amplex ${ }^{\circledR}$ Red reagent was added into the water right after the plasma treatment, and it reacted with $\mathrm{H}_{2} \mathrm{O}_{2}$ in a 1:1 stoichiometry to produce the red-fluorescent oxidation product, which was excited at $\lambda=550 \mathrm{~nm}$ and emitted at $\lambda=595 \mathrm{~nm}$. Similarly, Griess reagent was added into the water to detect nitrite, and the absorbance was measured at $\lambda=540 \mathrm{~nm}$. A nitrate reductase enzyme was used to transform nitrate into nitrite, and in that case the absorption spectral at $\lambda=540 \mathrm{~nm}$ reflected the total concentration of nitrite and nitrate. Then the nitrate concentration was obtained by using such total concentration minus the nitrite concentration. For the measurement of $\mathrm{O}_{3}$, an indigo reagent (AccuVac ${ }^{\circledR}$ Ozone Reagent) was utilized, and the absorption spectral at $\lambda=600 \mathrm{~nm}$ was recorded by a spectrophotometer (Hach, DR3900). The offline measurements were also performed for similar deionized water without the cover of gelatin gels, of which the results were used as control groups to reflect the barrier effects of the gelatin gels. All the offline measurements were repeated three times, except for the $\mathrm{H}_{2} \mathrm{O}_{2}$ permeability test which was repeated five times for each DC voltage to facilitate statistical analysis.

Furthermore, small electric fields were applied on the gelatin gels and the gelatin gel-covering deionized water as described above, for the purpose of controlling the penetration of RONS. The experimental conditions and the measurement methods are similar as described above, only that two extra electrodes were used. A grounded wire-mesh was put on the gelatin gels, and a low-voltage plane electrode placed underneath the dish. Small voltages of $\pm 5, \pm 10, \pm 15$ and $\pm 20 \mathrm{~V}$ were applied on the low-voltage plane electrode, and therefore an electric field of less than $20 \mathrm{~V} \mathrm{~cm}^{-1}$ was applied on the gelatin gels as well as the deionized water. The penetration depth of nitrite in the gelatin gels and the concentration of $\mathrm{H}_{2} \mathrm{O}_{2}$ in the deionized water were measured. Their dependence on the

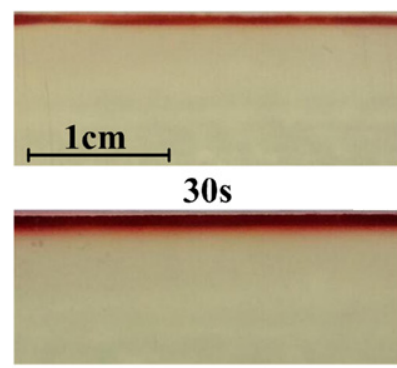

120s

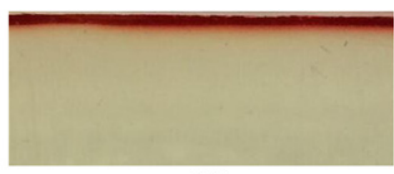

$60 \mathrm{~s}$

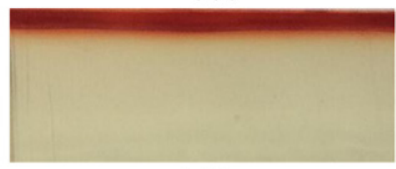

240s
Figure 2. Side images of gelatin gels mixed with Griess reagent after different times of plasma treatment.

small voltage value indicates the effect of electric field on the permeability of gelatin gels.

\section{Results and discussions}

\subsection{Penetration processes of nitrite in gelatin gels}

The side images of gelatin gels mixed with Griess reagent in quartz dishes are shown in figure 2, with respect to plasma treatment times of $30,60,120$ and $240 \mathrm{~s}$. The mass fraction of gelatin is $15 \%$. It can be seen that the depth of dark reddyed area (representing the existence of nitrite) increases with the plasma treatment time, indicating that a large amount of nitrite species diffuse across the air gap and keep penetrating into the gelatin gels. The boundaries of dyed and un-dyed areas are parallel to the gas-gelatin gel interface, indicating that the particle flux of nitrite on the gelatin gel surface is homogeneous. Compared to a similar experimental system where a dish of water is treated by surface air discharge [13], such boundaries are much more smooth and clear, because the gelatin inhibits the liquidity of water and the irregular activity of aqueous molecules. This allows the penetration depths of nitrite to be accurately determined in real time.

As presented above, absorption spectroscopy is utilized for determining the penetration depths of nitrite in the gelatin gels. The typical absorption spectra in the visible light range are shown in figure 3(a) for a gelatin gel (5\% gelatin in mass) 

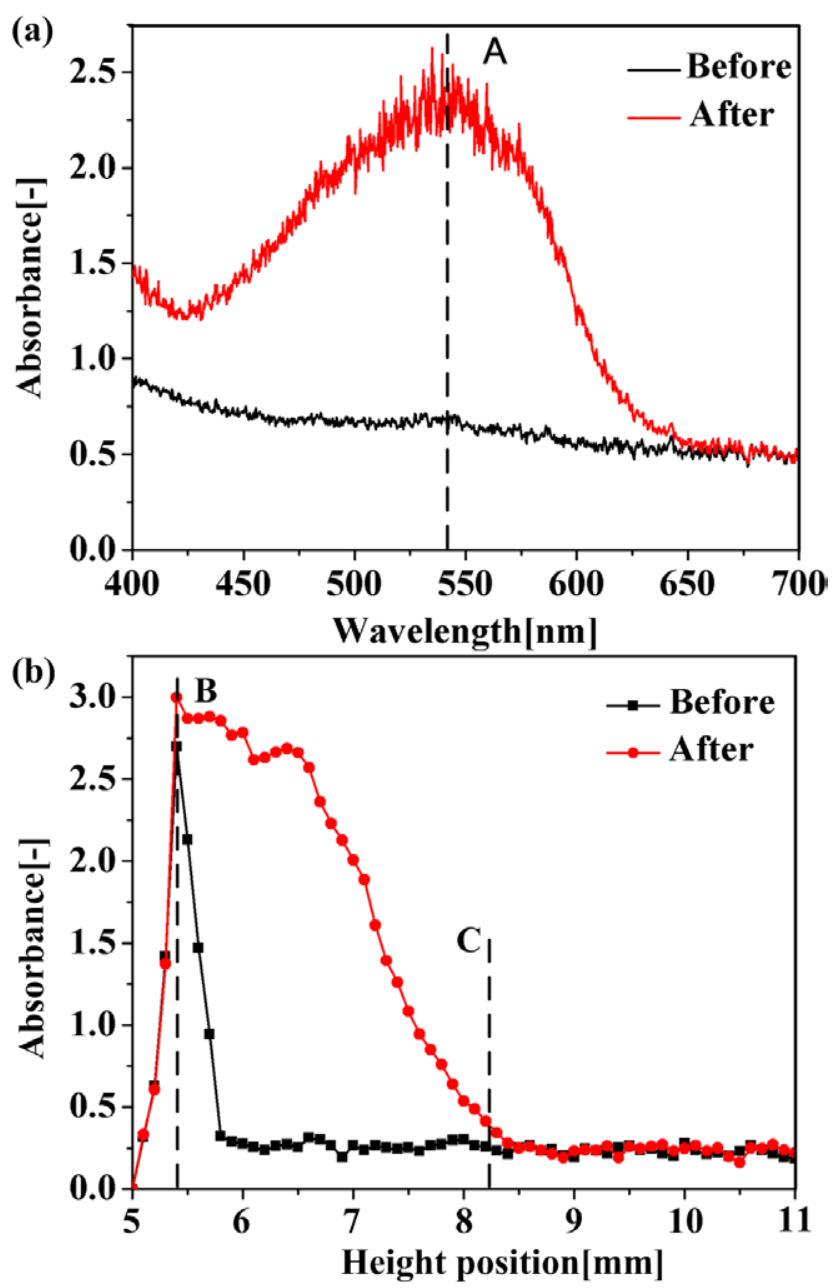

Figure 3. Absorption spectral image in the visible range (a) and absorbance at $\lambda=540 \mathrm{~nm}$ for different height positions (b) of gelatin gels before and after 5 min plasma treatment.

before and after $5 \mathrm{~min}$ plasma treatment. For the measurements in the figure, the line light is incident on a dish side, $5 \mathrm{~mm}$ from and parallel to the upper surface of the dish, and its optical distance in the gelatin gel is $2 \mathrm{~cm}$. It can be seen that the difference in absorbance is maximum at $\lambda=540 \mathrm{~nm}$ (marked A in figure 3(a)), indicating that this wavelength is most sensitive to the existence of nitrite. So, in figure 3(b) this wavelength is used to determine the penetration depth of nitrite for a plasma treatment time of $5 \mathrm{~min}$. For the gelatin gel before and after plasma treatment, both absorbance curves have peaks at a height position of $\sim 5.4 \mathrm{~mm}$ (scale in the height adjuster) which are marked B in figure 3(b). This is caused by the diffraction and interference of light at the gas-gelatin gel interface, which cannot be eliminated even when two field stops were used. The absorbance curves coincide when the height position is larger than $8.5 \mathrm{~mm}$, indicating that little nitrite exists in the gelatin gels beyond such a height value. The dash line marked with $\mathrm{C}$ in figure 3 (b) represents the height position where the difference in absorbance of the two curves is 0.1 . This difference value definitely demonstrates the existence of nitrite in the gelatin gel, and it is large enough to avoid the probable disturbance. The density of nitrite at position $\mathrm{C}$ for the plasma treated gelatin gel is about $3 \times 10^{18} \mathrm{~cm}^{-3}$
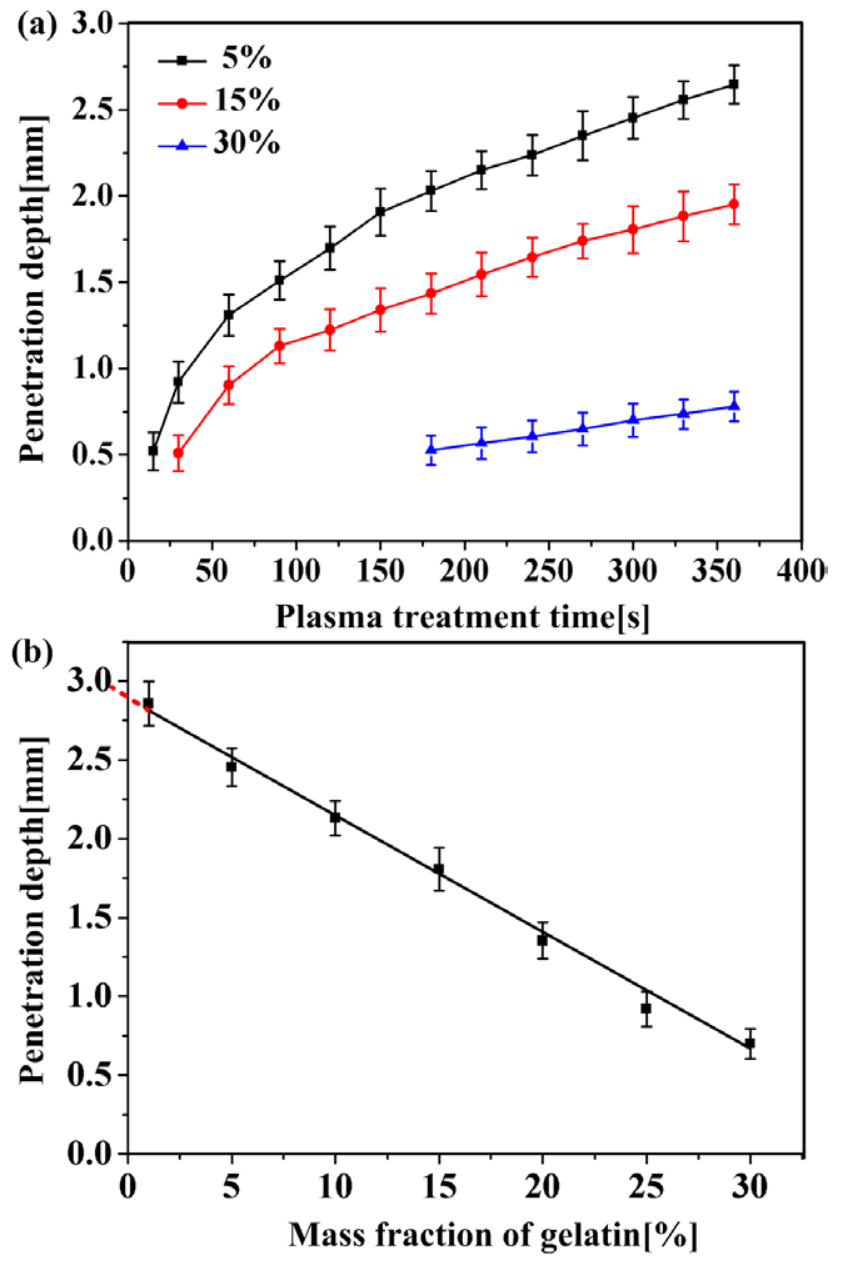

Figure 4. Penetration depths of nitrite with plasma treatment time (a) and their dependence on the mass fraction of gelatin (b).

( $\sim 5 \mathrm{nM})$, by comparing with the absorbance of a gelatin gel made by mixing gelatin powder with $\mathrm{NaNO}_{2}$ solution. In this paper, the penetration depth is defined by using the height position of $\mathrm{C}$ minus that of $\mathrm{B}$.

The dependences of penetration depths of nitrite on the plasma treatment time are shown in figure 4(a), with respect to the gelatin gels with $5 \%, 15 \%$ and $30 \%$ mass fraction of gelatin. The data of penetration depths used in figure 4(a) are larger than $0.5 \mathrm{~mm}$, because below such a value the disturbance by diffraction and interference is strong (see figure 3(b)). It is interesting that the penetration depths for all three gelatin gels increase almost linearly when the treatment time is larger than $175 \mathrm{~s}$, but below such a value their increase rates keep decreasing. This trend is in accordance with the numerical results of RONS penetration in deionized water induced by a similar surface air discharge, in which the reason is mainly ascribed to the accumulation of RONS in the gas phase [11]. This applies to the gelatin gels since they are water-rich. A time of $\sim 175 \mathrm{~s}$ is needed for the gaseous RONS to accumulate to certain density values to reach equilibrium with the dissolution loss. It can be seen from figure 4(a) that the penetration depth decreases with the increasing mass fraction of gelatin, which is clearly shown in figure 4(b) for the plasma treatment time of $5 \mathrm{~min}$ with mass fraction of gelatin varying from $1.5 \%$ 
(a)

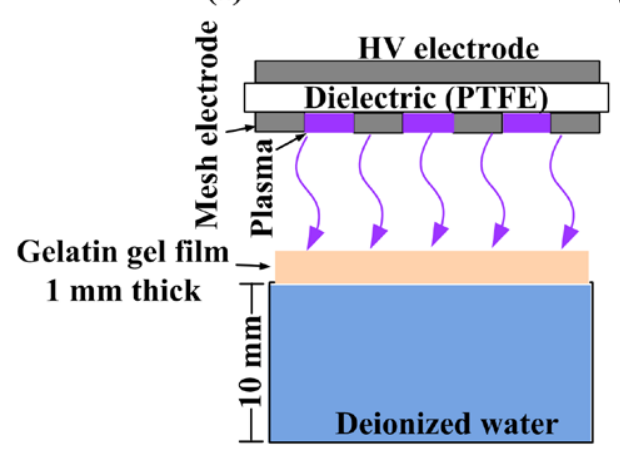

(b)

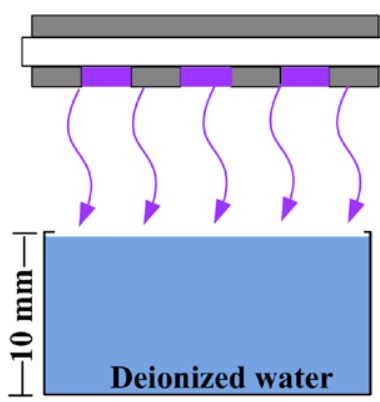

(c)

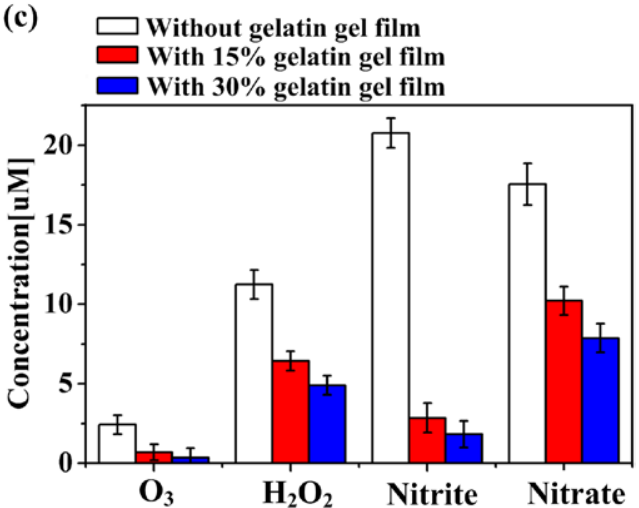

Figure 5. Deionized water treated by surface air discharge with the barrier of a gelatin gel film (a) and without the barrier of gelatin gel (b), as well as the RONS concentrations in deionized water for three experimental scenarios after 5 min plasma treatment (c).

to $30 \%$. Interestingly, the penetration depth decreases almost linearly with the increasing mass fraction of gelatin. Defining $c_{\mathrm{m}}(\%)$ as the mass fraction of water (one minus the mass fraction of gelatin) and $t_{\mathrm{p}}(\mathrm{s})$ the plasma treatment time, the penetration depth $d_{\mathrm{p}}(\mathrm{mm})$ can be roughly given by

$$
d_{\mathrm{p}}=2.25 \times 10^{-2}\left(c_{\mathrm{m}}-0.6\right) t_{\mathrm{p}}
$$

The formula (1) is valid for the mass fraction of water down to $70 \%$. Using this formula, the penetration speed of nitrite in pure water can be estimated to be $9 \times 10^{-3} \mathrm{~mm} \mathrm{~s}^{-1}$ with threshold nitrite concentration of $3 \times 10^{18} \mathrm{~cm}^{-3}(\sim 5 \mathrm{nM})$. This penetration speed is hard to measure as discussed above, but here we suggest that it can be estimated according to the penetration depth measurements for gelatin gels. Also, the penetration speeds of RONS in living tissues with different water content can be estimated according to this formula, which is important for plasma medicine research but has never been quantified.

\subsection{Permeability of RONS through the gelatin gels: quantification and control}

The main RONS in liquids induced by the surface air discharge are $\mathrm{O}_{3}, \mathrm{H}_{2} \mathrm{O}_{2}$, nitrite and nitrate as reported in the literature $[11,17,19]$. This might apply to the gelatin gels since they are water-rich. However, the penetrations of these RONS except for nitrite in gelatin gels are difficult to online measure by absorption spectroscopy. For example, the colorforming indicators for $\mathrm{H}_{2} \mathrm{O}_{2}$, potassium titanium (IV) oxalate and titanium (IV) oxysulfate $\left(\mathrm{TiOSO}_{4}\right.$ solution), were tried, but the absorption spectra of their products are close to that of the gelatin gels, and therefore the penetration depth cannot be determined explicitly. Instead, these RONS were detected in a dish of deionized water covered by gelatin gel films after plasma treatments, as shown in figure 5(a). For comparison, these RONS were also detected in a similar dish of deionized water without the covering of gelatin gel films, as shown in figure 5(b). The RONS concentrations of these three scenarios are shown in figure 5(c) for the plasma treatment time of $5 \mathrm{~min}$, of which the concentration differences reflect the barrier effects of the gelatin gels. The height of each bar represents the average measured concentration with an error bar of the standard derivation. The standard derivations are comparatively small and hence the concentration trends for the three scenarios are valid.

As shown in figure 5(c), the concentrations of all these RONS are significantly reduced by the barrier of the gelatin gel slice. For the $1 \mathrm{~mm}$ gelatin gel film with $15 \%$ gelatin in mass fraction, the concentration of $\mathrm{O}_{3}$ is reduced $\sim$ threefold, for $\mathrm{H}_{2} \mathrm{O}_{2}$ it is $\sim$ two- fold, for nitrite it is eight-fold, and for nitrate it is $\sim$ two-fold. When the mass fraction of gelatin increases to $30 \%$, the decrements are even higher by at least $30 \%$. It should be noted that the specificity of $\mathrm{H}_{2} \mathrm{O}_{2}$ indicator, Amplex ${ }^{\circledR}$ Red reagent, was tested by adding it into $\mathrm{NaNO}_{2}$ and $\mathrm{NaNO}_{3}$ solutions, of which no fluorescence signal was detected. It was reported that $\mathrm{O}_{3}$ has little influence on the Amplex ${ }^{\circledR}$ Red reagent [30-32], and even if it has, the measured concentrations of $\mathrm{H}_{2} \mathrm{O}_{2}$ will not change a lot since $\mathrm{O}_{3}$ densities are much lower. As shown in figure 5(c), the barrier effects of gelatin gels are different for the four RONS, which may be attributed to the RONS-incorporated chemical reactions that happen in the gelatin gels. For example, the reaction between $\mathrm{O}_{3}$ and $\mathrm{NO}_{2}^{-}$was predicted to be strong in liquids [11], as given by

$$
\mathrm{O}_{3}+\mathrm{NO}_{2}^{-} \rightarrow \mathrm{O}_{2}+\mathrm{NO}_{3}^{-} .
$$

Since gelatin gels are water-rich, this reaction may explain why the reductions of $\mathrm{O}_{3}$ and nitrite are stronger than that for $\mathrm{H}_{2} \mathrm{O}_{2}$ and nitrate. It should be noted that only four species are studied, and possibly the barrier effects for some other species have different trends.

From an applications point of view, it is of importance to enhance the permeability of living tissues because the target spots of many diseases are located in the living tissues with certain depths. Here small electric fields were used to regulate the permeability of RONS, which are capable of changing the penetration depths of $\mathrm{O}_{2}^{-}$and $\mathrm{H}_{2} \mathrm{O}_{2}$ in plasma-activated water as reported previously [33]. A grounded wire-mesh was put on the gelatin gels, and another plane electrode is put underneath the dish for which DC voltages of $\pm 5, \pm 10, \pm 15$ and $\pm 20 \mathrm{~V}$ were applied. For clinical applications, these DC voltages are in the safety range according to IEC/TS 61201-2007. The dish was either filled with gelatin gels for studying the regulation on the permeability of nitrite (see figure 6(a)), or with 
(a)

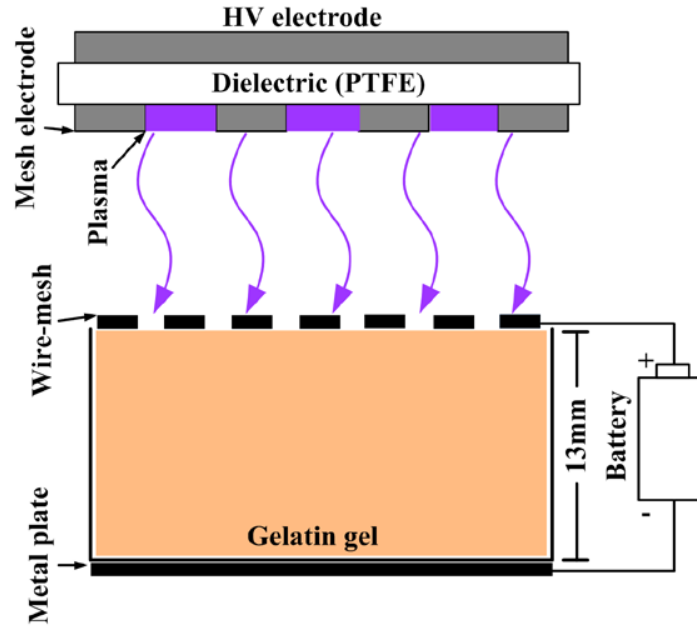

(d)

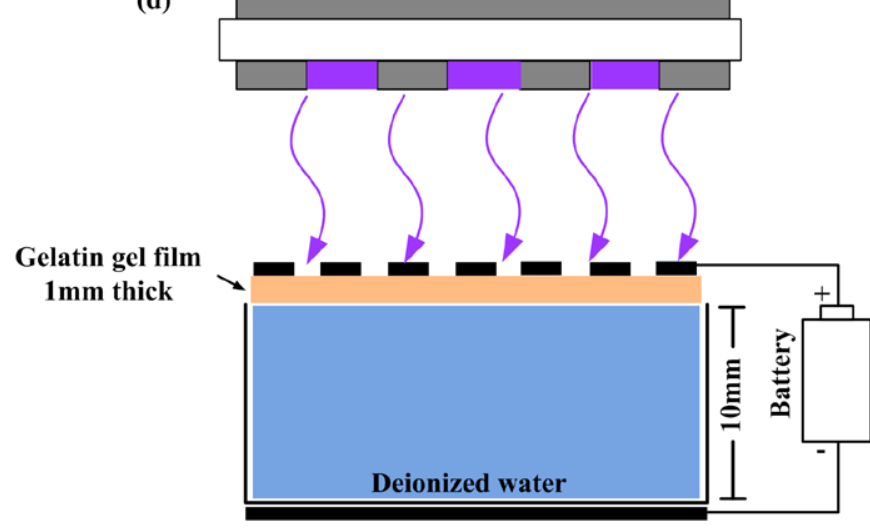

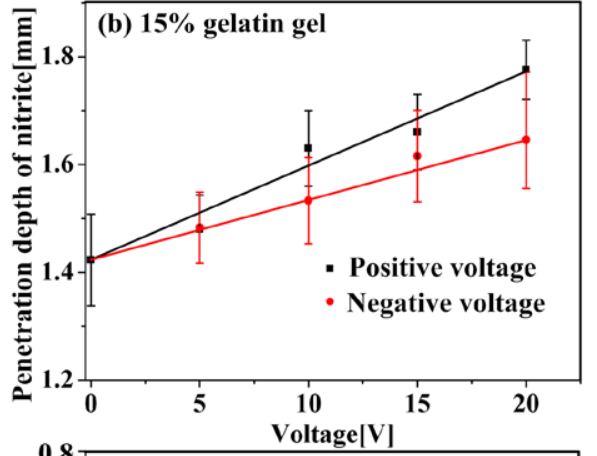
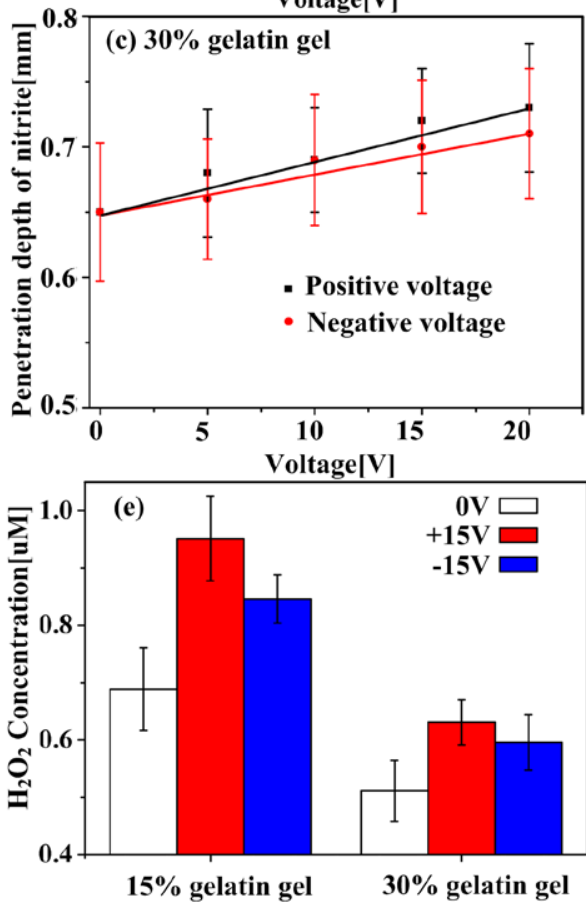

Figure 6. The influence of small electric fields on the permeability of RONS. (a), (d) Schematic of the experimental setup; (b), (c) penetration depths of nitrite in gelatin gels for different operating DC voltages; (e) concentrations of $\mathrm{H}_{2} \mathrm{O}_{2}$ in deionized water for different operating DC voltages. The time for surface discharge treatment is $5 \mathrm{~min}$.

deionized water covered by a $1 \mathrm{~mm}$ thick gelatin gel film for that of $\mathrm{H}_{2} \mathrm{O}_{2}$ (see figure 6(d)). The time for plasma treatment was kept at $5 \mathrm{~min}$.

As shown in figures 6(b) and (c), the penetration depth of nitrite in gelatin gels (with $15 \%$ and $30 \%$ mass fraction of gelatin) increases linearly with the electric field regardless of its direction. For the positive voltage (the electric field is upwards) this increase is easy to understand at first glance because the electric field force for $\mathrm{NO}_{2}^{-}$is downwards, but for the negative voltage (the electric field is downwards) it is very strange since the force repels $\mathrm{NO}_{2}^{-}$for penetration. Comparing the positive voltage with the negative one, the former has a stronger effect on increasing the penetration of nitrite. These phenomena suggest that the direct drive on $\mathrm{NO}_{2}^{-}$by the electric field force might be one reason for enhancing its penetration depth, but it should not be the dominant one.

As shown in figure $6(\mathrm{e})$, the concentration of $\mathrm{H}_{2} \mathrm{O}_{2}$ in the gelatin gel-covering water is also enhanced by the electric field, and this enhancement is regardless of the electric field direction too. Here, the measurements were repeated five times for each DC voltage, and an independent-samples $\mathrm{T}$ test was made on the results of different DC voltages as shown in figure 6(e), which in general demonstrates significant variations among the cases of $0 \mathrm{~V},+15 \mathrm{~V}$ and $-15 \mathrm{~V}$ $(p<0.05)$. Only when the mass fraction of gelatin gel is $30 \%$ do the results of $+15 \mathrm{~V}$ and $-15 \mathrm{~V}$ have no significant differences $(p \sim 0.25)$. Since $\mathrm{H}_{2} \mathrm{O}_{2}$ is a neutral species, this further confirms that the electric field force on the objective RONS should not be the main mechanism for the increase of gelatin gel permeability. According to the simulation for the plasma-activated water as presented previously, the eigenelectric field formed by the self-ionization of RONS (mainly $\mathrm{HNO}_{2}, \mathrm{HNO}_{3}$ and $\mathrm{N}_{2} \mathrm{O}_{5}$ ) will inhibit their penetration [11]. So, one hypothesis is that the extra electric field destroys the hydrolysis-formed electric field and therefore enhances the permeability. Another hypothesis is the structures of the tissue models are changed by the electric field, although no change is observed. This is possible because the comparable electric fields have been found to enhance drug delivery in human skin $[34,35]$. Therefore, the results shown in figure 6 suggest that small electric fields of several to several tens of $\mathrm{V} \mathrm{cm}-1$ are effective for changing the living tissue permeability; values much lower than that needed for cell electroporation [36]. 


\section{Conclusion}

Delivery of plasma-induced RONS to target spots in human tissues is crucial for plasma medicine, but so far the barrier effects of the tissues have not been well understood. Here, gelatin gels are used as surrogates of human tissues to study their barrier effects on the incoming RONS generated by a surface air discharge. The penetration processes of nitrite into the model tissue are measured during plasma treatment by using absorption spectroscopy and a color-forming indicator. The concentrations of nitrite, nitrate, $\mathrm{O}_{3}$ and $\mathrm{H}_{2} \mathrm{O}_{2}$ in the deionized water underneath $1 \mathrm{~mm}$ thick gelatin gel films are measured by spectrophotometry after plasma treatment, and the permeability of the tissue models is then quantified by comparing the concentrations with that without the covering of gelatin gel films. For the purpose of controlling the barrier effects of the tissue models, a small electric field of up to $20 \mathrm{~V} \mathrm{~cm}^{-1}$ is applied on the plasma-treated sample. In that case the penetration depths of nitrite as well as the concentrations of $\mathrm{H}_{2} \mathrm{O}_{2}$ in the deionized water are measured again, and the effect of electric field on the barrier effects is then obtained by comparing the results with that without the electric fields.

It is found that the penetration speed of nitrite decreases linearly with the mass fraction of gelatin in the tissue models. For mass fractions of water down to $70 \%$ and the threshold nitrite concentration of $3.01 \times 10^{18} \mathrm{~cm}^{-3}$, the penetration depth $(\mathrm{mm})$ can be formulated by $2.25 \times 10^{-2}\left(c_{\mathrm{m}}-0.6\right) t_{\mathrm{p}}$ with $c_{\mathrm{m}}(\%)$ the mass fraction of water and $t_{\mathrm{p}}(\mathrm{s})$ the plasma treatment time. This formula might be useful for roughly estimating the penetration depths of plasma-induced RONS in human tissues as well as biological solutions. The permeabilities of tissue models on nitrite, nitrate, $\mathrm{O}_{3}$ and $\mathrm{H}_{2} \mathrm{O}_{2}$ are different, of which they are smaller for nitrite and $\mathrm{O}_{3}$. This suggests that the composition of RONS in living tissues vary with the tissue depth and plasma treatment time, which should be considered for clinical applications. It is interesting that small electric fields can greatly reduce the barrier effects of the tissue models on nitrite and $\mathrm{H}_{2} \mathrm{O}_{2}$, which might be useful for the delivery of RONS to target spots in human tissues. This reduction is relatively independent of the polarity of the electric field, suggesting that the electric field force on the object RONS should not be the dominant mechanism. Two hypotheses are made for this reduction: (1) the electric field formed by hydrolysis of plasma-induced species is destroyed by the external electric field which enhance the penetration speeds of RONS, and (2) the structure of gelatin gels might be changed to some extent by the electric field, although nothing is observed. Further studies are required to elucidate the underlying mechanism.

\section{Acknowledgments}

This work was supported by the National Science Foundation of China (Grant No. 51307134 and 51521065), the Fundamental Research Funds for the Central Universities and the State Key Laboratory of Electrical Insulation and Power Equipment (Grant No. EIPE14123).

\section{References}

[1] Fridman G, Friedman G, Gutsol A, Shekhter A B, Vasilets V N and Fridman A 2008 Applied plasma medicine Plasma Process. Polym. 5503

[2] Lloyd G, Friedman G, Jafri S, Schultz G, Fridman A and Harding K 2010 Gas plasmas: medical uses and developments in wound care Plasma Process. Polym. 7194

[3] Sladek R E J, Stoffels E, Walraven R, Tielbeek P J A and Koolhoven R A 2004 Plasma treatment of dental cavities: a feasibility study IEEE Trans. Plasma Sci. 321540

[4] Pouvesle J M et al 2015 Potential of plasma based soft and/ or combined cancer treatments Proc. 2nd Int. Workshop on Plasma for Cancer Treatment (Nagoya, Japan) (https://hal. archives-ouvertes.fr/hal-01148938/)

[5] Kong M G, Kroesen G, Morfill G, Nosenko T, Shimizu T, van Dijk J and Zimmermann J L 2009 Plasma medicine: an introductory review New J. Phys. 11115012

[6] Breslow A 1970 Thickness, cross-sectional areas and depth of invasion in the prognosis of cutaneous melanoma Ann. Surg. 172902

[7] Clark W H, From L, Bernardino E A and Mihm M C 1969 The histogenesis and biologic behavior of primary human malignant melanomas of the skin Cancer Res. 29705

[8] Chen C, Liu D X, Liu Z C, Yang A J, Chen H L, Shama G and Kong M G 2014 A model of plasma-biofilm and plasmatissue interactions at ambient pressure Plasma Chem. Plasma Process. 34403

[9] Gniadecka M, Nielsen O F and Wulf H C 2003 Water content and structure in malignant and benign skin tumours J. Mol. Struct. 661-2 405

[10] Tian W and Kushner M J 2014 Atmospheric pressure dielectric barrier discharge interacting with liquid covered tissue J. Phys. D: Appl. Phys. 47165201

[11] Liu Z C, Liu D X, Chen C, Li D, Yang A J, Rong M Z, Chen H L and Kong M G 2015 Physicochemical processes in the indirect interaction between surface air plasma and deionized water J. Phys. D: Appl. Phys. 48495201

[12] Rens J, Schoof J, Ummelen F, Vugt D, Bruggeman P and Veldhuizen E 2014 Induced liquid phase flow by RF Ar cold atmospheric pressure plasma jet IEEE Trans. Plasma Sci. 422622

[13] Oehmigen K, Hoder T, Wilke C, Brandenburg R, Hähnel M, Weltmann K and Woedtke T 2011 Volume effects of atmospheric-pressure plasma in liquids IEEE Trans. Plasma Sci. 392646

[14] Marshall S E, Jenkins A T A, Al-Bataineh S A, Short R D, Hong S H, Thet N T, Oh J-S, Bradley J W and Szili E J 2013 Studying the cytolytic activity of gas plasma with self-signalling phospholipid vesicles dispersed within a gelatin matrix J. Phys. D: Appl. Phys. 46185401

[15] Szili E J, Bradley J W and Short R D 2014 A 'tissue model' to study the plasma delivery of reactive oxygen species J. Phys. D: Appl. Phys. 47152002

[16] Gaur N, Szili E J, Oh J S, Hong S H, Michelmore A, Graves D B, Hatta A and Short R D 2015 Combined effect of protein and oxygen on reactive oxygen and nitrogen species in the plasma treatment of tissue Appl. Phys. Lett. 107103703

[17] Pavlovich M J, Chang H-W, Sakiyama Y, Clark D S and Graves D B 2013 Ozone correlates with antibacterial effects from indirect air dielectric barrier discharge treatment of water J. Phys. D: Appl. Phys. 46145202

[18] Zhong S Y, Dong Y Y, Liu D X, Xu D H, Xiao S X, Chen H L and Kong M G 2015 Surface air plasma-induced cell death and cytokines release of human keratinocytes in the context of psoriasis Br. J. Dermatol. 174 542-52

[19] Oehmigen K, Hähnel M, Brandenburg R, Wilke Ch, Weltmann K-D and von Woedtke Th 2010 The role of 
acidification for antimicrobial activity of atmospheric pressure plasma in liquids Plasma Process. Polym. 7 250-7

[20] Olszewski P, Li J F, Liu D X and Walsh J L 2014 Optimizing the electrical excitation of an atmospheric pressure plasma advanced oxidation process J. Hazard. Mater. 279 60-6

[21] Szili E J, Oh J S, Hong S-H, Hatta A and Short R D 2015 Probing the transport of plasma-generated RONS in an agarose target as surrogate for real tissue: dependency on time, distance and material composition J. Phys. D: Appl. Phys. $\mathbf{4 8} 202001$

[22] Szili E J, Hong S-H and Short R D 2015 On the effect of serum on the transport of reactive oxygen species across phospholipid membranes Biointerphases 10029511

[23] Fridman G, Brooks A D, Balasubramanian M, Fridman A, Gutsol A, Vasilets V N, Ayan H and Friedman G 2007 Comparison of direct and indirect effects of non-thermal atmospheric-pressure plasma on bacteria Plasma Process. Polym. 4370

[24] Shimizu T, Sakiyama Y, Graves D B, Zimmermann J L and Morfill G E 2012 The dynamics of ozone generation and mode transition in air surface micro-discharge plasma at atmospheric pressure New J. Phys. 14103028

[25] Ellerweg D, Benedikt J, Keudell A V, Knake N and Gathen V S D 2010 Characterization of the effluent of a $\mathrm{He} / \mathrm{O}_{2}$ microscale atmospheric pressure plasma jet by quantitative molecular beam mass spectrometry New J. Phys. 12013021

[26] Li D, Liu D X, Nie Q Y, Li H P, Chen H L and Kong M G 2014 Array of surface-confined glow discharges in atmospheric pressure helium: modes and dynamics Appl. Phys. Lett. 104204101

[27] Li D, Liu D X, Nie Q Y, Xu D H, Li Q S and Kong M G 2014 Emission characteristics of surface micro-discharge in atmospheric-pressure $\mathrm{He} / \mathrm{N}_{2}$ mixture IEEE Trans. Plasma Sci. 422640
[28] Li D, Liu D X, He T T, Li Q S, Wang X H and Kong M G 2015 Three distinct modes in a surface micro-discharge in atmospheric pressure $\mathrm{He}+\mathrm{N}_{2}$ mixtures Phys. Plasmas 22123501

[29] Davis F E, Kenyon K and Kirk J 1953 A rapid titrimetric method for determining the water content of human blood Science 118276

[30] Jablonowski H, Hänsch M A C, Dünnbier M, Wende K, Hammer M U, Weltmann K-D, Reuter S and Woedtke T V 2015 Plasma jet's shielding gas impact on bacterial inactivation Biointerphases 10029506

[31] Winter J, Wende K, Masur K, Iseni S, Dünnbier M, Hammer M U, Tresp H, Weltmann K-D and Reuter S 2013 Feed gas humidity: a vital parameter affecting a cold atmospheric-pressure plasma jet and plasma-treated human skin cells J. Phys. D: Appl. Phys. 46295401

[32] Yamashita K, Miyoshi T, Arai T, Endo N, Itoh H, Makino K, Mizugishi K, Uchiyama T and Sasada M 2008 Ozone production by amino acids contributes to killing of bacteria Proc. Natl Acad. Sci. USA 105 16912-7

[33] Xu D H, Liu D X, Wang B Q, Chen C, Chen Z Y, Li D, Yang Y J, Chen H L and Kong M G 2015 In situ OH generation from and $\mathrm{H}_{2} \mathrm{O}_{2}$ plays a critical role in plasma induced cell death PLoS One 6 e0128205

[34] Kalia Y N, Naik A, Garrison J and Guy R H 2004 Iontophoretic drug delivery $A d v$. Drug Delivery Rev. 56 619-58

[35] Marro D, Guy R H and Delgado-Charroa M B 2001 Characterization of the iontophoretic permselectivity properties of human and pig skin J. Control. Release $70213-7$

[36] Babaeva N Y and Kushner M J 2010 Intracellular electric fields produced by dielectric barrier discharge treatement of skin J. Phys. D: Appl. Phys. 43185206 